\title{
The Analysis of Mobile WiMAX and LTE using MIMO technique \\ ${ }^{1}$ Roopali Garg, ${ }^{2}$ Shafi Singla \\ ${ }^{1}$ Coordinator deptt. (IT), Assistant Professor \\ UIET,Panjab University, Chandigarh \\ roopali.garg@gmail.com \\ ${ }^{2}$ Research Scholar(IT) \\ UIET, Panjab University, Chandigarh,India \\ shif.singla@gmail.com
}

\begin{abstract}
A quality of service is a fundamental component of the $4 G$ broadband network for satisfactory service delivery by evolving internet application to end user, and managing the network resources. The 4G technology has emerged one of the most fruitful technologies as it supports large number of applications including VOIP, video conferencing, file transfer, video streaming and web browsing. IEEE 802.16E, IEEE 802.16M and LTE along with various application and QoS requirement also support Multiple-Input-multiple-output (MIMO) techniques including Spatial Multiplexing (SM), Space Time Block Coding(STBC) and Eigen Beam-forming (BM).In the paper we will study Physical, MAC layer of WiMAX and LTE. We will also study MIMO techniques. In this paper the detailed study of the throughput for a MIMO mobile WiMAX system and LTE under two different PHY PER QOS threshold. Various modulation and channel codinng techniques have been applied.
\end{abstract}

Index terms: MOBILE WIMAX, LTE, PHY, MAC, MIMO,

\section{INTRODUCTION}

Quality of service (QoS) is becoming significant as broadband subscribers and traffic volume per subscriber is increasing day by day. Fourth-generation (4G) broadband wireless technologies such as IEEE $802.16 \mathrm{e}$, JEEE $802.16 \mathrm{~m}$, and Third Generation Partnership Project (3GPP) Long Term Evolution (LTE) have been designed to support various mobile and internet applications. The first WiMAX systems were based on the IEEE 802.16-2004 standard [1]. This targeted fixed broadband wireless Application via the installation of Customer Premises Equipment (CPE)

Mobile WiMAX extends the original OFDM PHY layer to support multiple-access by using scalable OFDMA [2].DataStream to and from individual user is multiplexed for both Uplink (UP) and Downlink(DL) to groups of subchannels. By adopting a scalable PHY architecture, mobile WiMAX is able to support wide range of bandwidth.

The combination of MIMO and OFDMA technique achieve higher spectral efficiency and link reliability without additional bandwidth or transmit power. $4 G$ technologies supports full range of smart antenna techniques including Alamouti spatial transmit diversity [3],spatial multiplexing, and closed-loop MIMO eigen-beamforming (EB) [5].TCP and UDP are the two main protocols that have different QoS criteria and are used in different application. While TCP supports web browsing, emails and FTP, UDP supports video streaming.

The long-term evolution (LTE) as defined by the 3rd Generation Partnership Project (3GPP) is a highly flexible radio interface [8]. The LTE provides peak rates of $300 \mathrm{Mb} / \mathrm{s}$, a radio-network delay of less than $5 \mathrm{~ms}$, a significant increase in spectrum efficiency compared to previous cellular systems, and a new flat radio-network architecture designed to simplify operation and to reduce cost. The basic transmission schemes in uplink and downlink are described along with hybrid $A R Q$, multiple-antenna transmission techniques.

This paper further studies in detail how the difference in PHY requirements, modulation leads to difference in throughput. This paper is organized as follows: Section II contains Mobile WiMAX MAC layer description. Next, Section III is about PHY layer description. Section IV. is about MAC and PHY layer of LTE, Different MIMO techniques are discussed in section V. Section VI contains conclusion and the last section VII contains references.

\section{MOBILE WIMAX MAC LAYER DESCRIPTION}

The Medium Access Control (MAC) layer of WiMAX is divided into three sublayers: the service-specific Convergence Sublayer (CS), the common-part sublayer (CPS), and the Security Sublayer (SS) which handles authorization, encryption and is also involved in proper exchange of encryption keys between BS and MS.

The convergence sublayer is responsible for performing all the operations that are dependent on higher layer and receive packets known as MAC service data units(SDU).MAC layer is also responsible for identifying a logical connection by using a unidirectional connection identifier(CID) between Base station (BS) and Mobile station(MS).CS performs operations such as header compression which is done by removing the repetitive part of header of each SDU at the transmitter end and inserting those parts again into the SDU at receiver end before delivering them to higher layer. QoS requirement for MAC-PDU belonging to different service flow is provided for UP and DL traffic as a key concept of QoS architecture. Each Service flow have a service flow ID(SFID) and connection ID(CID).Various parameters including Jitter, data rate, packet error rate, system availability are tied to overall QoS requirement. Scheduling services define the mechanisms used to allocate resources to different applications. Various scheduling services namely Unsolicited Grant Service(UGS), The real time Polling Service (rtPS). Non real time Polling Service (nrtPS), The Best Effort (BE) and the extended real time Polling Service (ertPS). These are designed for constant bit rate (CBR) real-time, variable bit rate (VBR) real-time, VBR non real-time, and BE applications[4]. 
The common part sublayer operations are independent of the higher layer scheduling, bandwidth allocation, AutomaticRepeat-request (ARQ), modulation and construction of MAC Packet Data Unit (PDUs) by fragmentation and concatenation of SDU. Using the physical effectively multiple MAC-PDUs are concatenated when destined for same receiver.

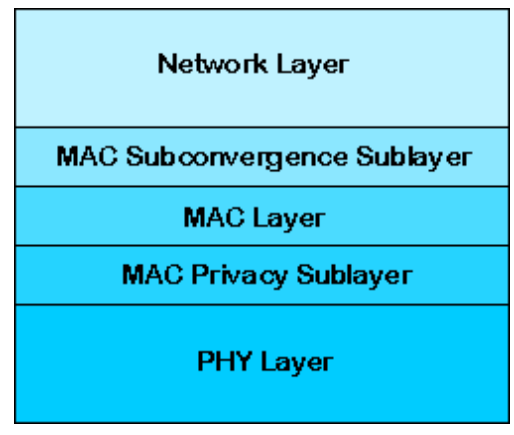

Fig 1 WiMAX MAC architecture

For non-ARQ-enabled connections, each fragment of the SDU is transmitted in sequence.ARQ enabled connections , SDU is first partitioned into ARQ blocks and a block sequence number (BSN) is assigned to each ARQ block. The length of $A R Q$ is specified by the BS for each CID, using the ARQ BLOCK- SIZE parameter. Once the SDU is partitioned into $A R Q$ blocks, the partitioning remains in effect until all the $A R Q$ blocks have been received and acknowledged by the receiver. After the ARQ block partitioning, the SDU is assembled into MAC-PDUs.

\section{MOBILE WIMAX PHY LAYER DESCRIPTION}

The mobile WiMAX standard builds on the principles of OFDM by adopting a Scalable OFDMA-based PHY layer (SOFDMA). FFT size is scalable with bandwidth that means when the available bandwidth increases, the FFT size is also increased such that the subcarrier spacing is always $10.94 \mathrm{kHz}$. This ensures a fixed OFDMA symbol duration. Since the basic resource unit (i.e. the OFDMA symbol duration) is fixed, the impact of bandwidth scaling is minimized to the upper layers. Parameters of OFDMA PHY are described in Table 1

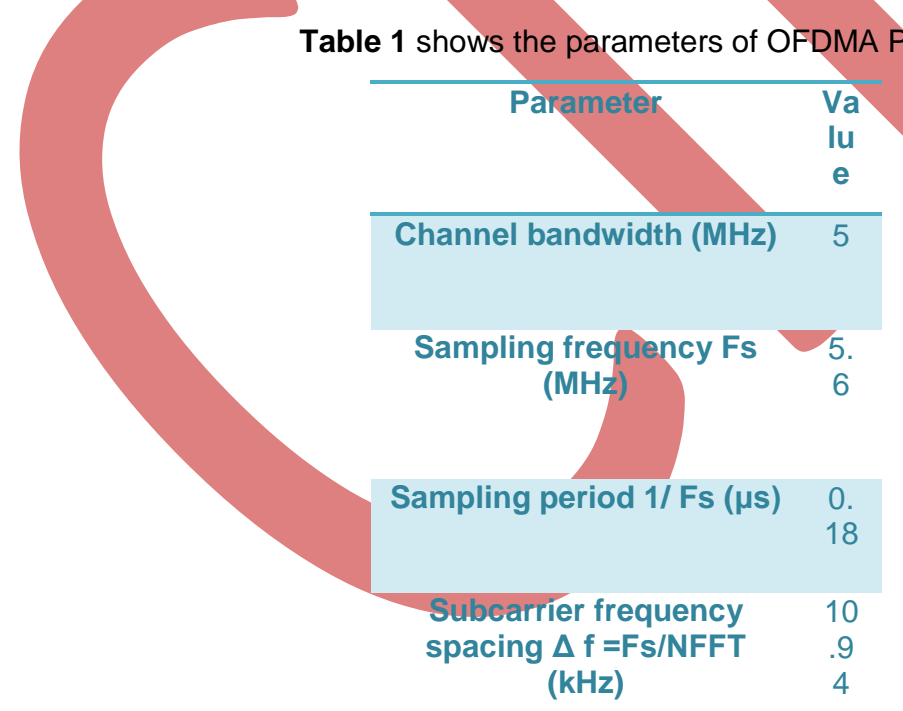

\begin{tabular}{cc}
\hline Useful symbol period Tb $=$ & 91 \\
$\mathbf{1} / \boldsymbol{\Delta} \mathbf{f}(\boldsymbol{\mu s})$ & .4 \\
OFDMA symbol duration & 10 \\
Ts $=\mathrm{Tg}+\mathrm{Tb}(\boldsymbol{\mu s})$ & 2. \\
& 9 \\
Number of used & 42 \\
subcarriers (Nused) & 1 \\
Number of pilot & 60 \\
subcarriers & \\
Number of data & 36 \\
subcarriers & 0 \\
\hline
\end{tabular}




\begin{tabular}{cc}
\hline $\begin{array}{c}\text { Number of data } \\
\text { subcarriers in each } \\
\text { subchannel }\end{array}$ & 24 \\
\hline $\begin{array}{c}\text { Number of subchannels } \\
\text { Number of users (Nusers) }\end{array}$ & 3 \\
\hline $\begin{array}{c}\text { Number of subchannels } \\
\text { allocated to each user } \\
\text { (NALSU) }\end{array}$ & 5 \\
\hline
\end{tabular}

In IEEE 802.16e-2005, WiMAX function stages are illustrated in Fig.2. The channel coding stage includes randomization convolution encoding (native code rate is $1 / 2$ ) and puncturing to produce higher code rates. Next is interleaving which is performed independently on each FEC block. It is mainly used to minimize the impact of bursts error. Next is modulation that is done using different scheme like 16QAM, 64QAM, QPSK etc.

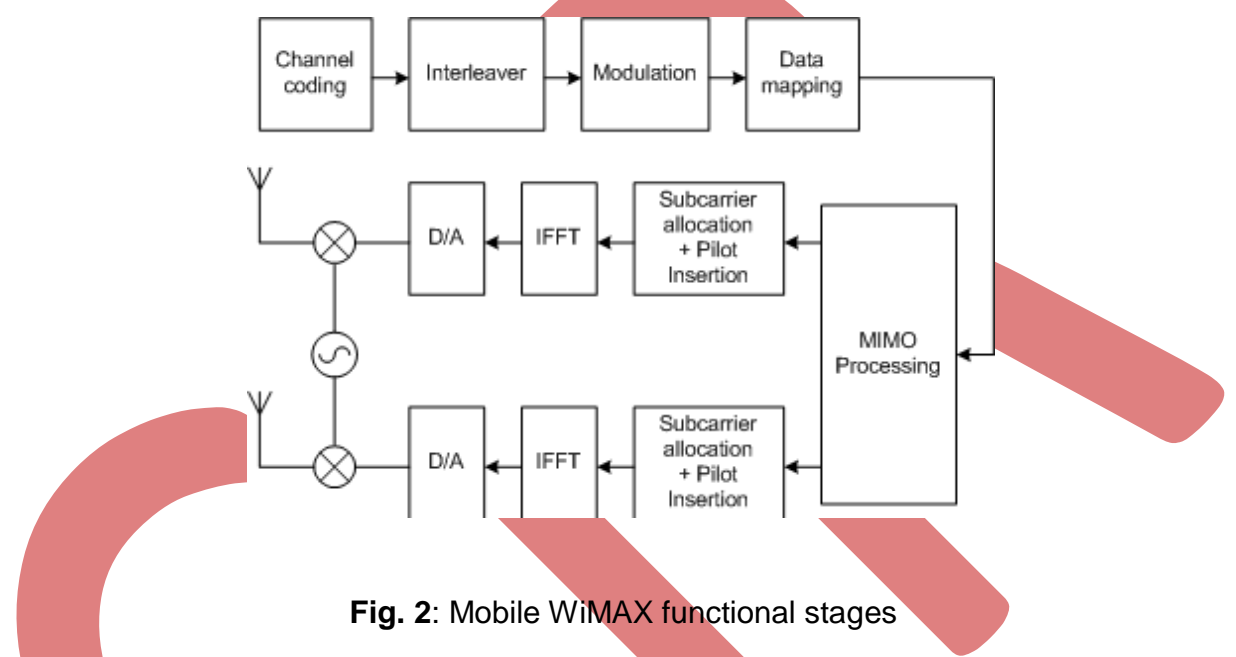

The data is then mapped by segmenting the sequence of modulation symbols into a sequence of slots (the minimum data allocation unit) and then mapping these slots into a data region. In last step, space time encoder is used to encode data into different smart antenna techniques.

\section{LTE PHY AND MAC LAYER DESCRIPTION}

In LTE, the Medium Access Control (MAC) layer handles logical-channel multiplexing, hybrid-ARQ retransmissions, and uplink and downlink scheduling. The MAC offers services to the radio link control layer (RLC) in the form of logical channels. A logical channel is defined by the type of information it carries. Logical channels are basically control channels and transport channel are used for carrying user data. Uplink and Downlink scheduling decisions can be taken independently of each other within some limits that is set by UL/DL split in case of TDD operation). The scheduler is part of the MAC layer and controls the assignment of UL and DL resources and is also responsible for selecting the transportblock size, the modulation scheme, and the antenna mapping in case of multi-antenna transmission.

Downlink scheduling has one of the basic principles of shared-channel transmission on Downlink Shared Channel (DL-SCH) and Uplink Shared Channel (UL-SCH). Both transport channels used for uplink and downlink data in LTE supports hybrid ARQ, spatial multiplexing dynamic rate adaptation, channel-dependent scheduling in the time and frequency domains. The downlink scheduler is used to dynamically determine, in each $1 \mathrm{~ms}$ interval, which terminal(s) that are supposed to receive DL-SCH transmission and on what resource. The basic time frequency unit in the scheduler is resource block. Information about the downlink channel conditions, necessary for channel dependent scheduling is supplied by channel-quality reports. The channel-quality report, also known as Channel-Quality Indicator (CQI).In the uplink, the terminals can be configured to transmit a sounding reference signal, the reception quality of which may be used for uplink channel-dependent scheduling.

LTE includes a two-layered retransmission scheme: hybrid ARQ located in the MAC layer and ARQ in the RLC layer. Low overhead feedback and support for soft combining with incremental redundancy is complemented by a highly reliable selective-repeat $A R Q$ is provided by lightweight hybrid $A R Q$ which handles most of the errors and also provide feedback to transmitter of each transmitted sub frame. The use of a two-layered mechanism achieves low latency and low overhead without sacrificing reliability.

The physical layer is responsible for coding, physical-layer hybrid-ARQ processing, modulation, multi-antenna processing, and mapping of the signal to the appropriate physical time-frequency resources. In the first step of the transport-channel processing, a Cyclic Redundancy Check (CRC) is calculated and appended to each 
transport block. The channel coding stage includes randomization, convolution encoding (turbo code $1 / 3$ ) and puncturing to produce higher code rates.

Next comes downlink physical-layer hybrid-ARQ, its functionality is to extract from the blocks of code bits delivered by the channel encoder, the exact set of bits to be transmitted within a given transmission time interval [8].

\section{MIMO SCENARIOS}

MIMO techniques have attracted a lot of attention in wireless communication as it offers significant increase in the coverage area, throughput and reliability without increasing the transmit power. In a flat fading channel, Where $P_{A}$ wants to send a message to $P_{B}, P_{A}$ has $N_{r}$ transmit antennas and $P_{B}$ has $N_{t}$ antennas then the complex channel matrix between $P_{A}$ and $P_{B}$ is $N_{r} \times N_{t}$ common represented by $H$.If $P_{A}$ sends $N_{t}$ dimensional signal $x$, then $P_{B}$ will receive $N_{r}$ Signal $y=H x+n$. Where $n$ is dimensional noise vector. In this paper we will discuss all the techniques including Spatial Multiplexing(SM), Space Time Block code(STBC) and Eigen beam forming that will use these principal and enhance communication and reliability.

A) Spatial Multiplexing: This technique transmit independent and separate encoded data signal from each of the multiple transmitter antennas. So space time dimension is used more than one time. If the transmitter is equipped with $N_{t}$ antennas and the receiver is equipped with $N r$ antennas. Then the maximum multiplexing is $N_{s} \neq N_{t} \times N_{r}$ where $N_{s}$ is no of signals that can be transmitted parallelly.

In LTE spatial multiplexing relies on codebook-based pre-coding, implying that for each combination of number of antennas NA and number of layers NL,A set of pre-coder matrices are defined by the specification. Based on measurements on the downlink reference signals of the different antennas, the mobile decides on a suitable rank (number of layers) and corresponding pre-coder matrix. This is then reported to the network. While a single rank, valid for the entire system bandwidth or multiple pre-coder matrices, valid for different parts of the system bandwidth, may be reported. The network takes this information into account but doesn't follow it as the network must explicitly signal what pre-coder matrices are used by means of the downlink control signaling. Though pre-coding can only be used for the DL-SCH transmission. In SM there are generally two codeword's where $\mathrm{NL} \geq 2$ and NA $\geq N L$. Taking a case where NL=3 and $\mathrm{NA}=4$. the first codeword is mapped by layer 1 and codeword is mapped by layer 2 and 3[8].

B) Space time block coding (STBC):Almouti scheme is used to implement STBC on downlink to transmit and receive diversity. Two transmit antennas and $\mathrm{M}$ receive antennas to provide a diversity order of $2 \mathrm{M}$ also called Almouti's 2 by 1

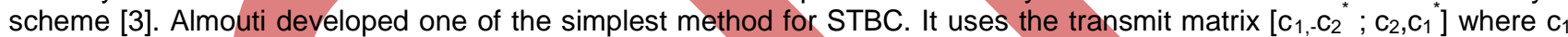
and $\mathrm{C}_{2}$ are OFDMA symbols. STBC Improves the reliability of data transmission by transmitting multiple copies of data stream to the receiver in a hope that some of them are received at a good state. Signal suffers from multipath fading and thermal noise so therefore it uses all the copies to extract the required information. Data is transmission into blocks, before data is transmitted these data blocks are distributed to multiple antennas. Newly derived Space-Time-Frequency codes (STFC) can be used to exploit both time as well as frequency diversity in the channel. The STFC showed diversity Gain compared to STC and SFC(space frequency code)[4].

c)Beam-forming(BM): It is a powerful technique for increasing the throughput and performance of wireless communication systems that employ multiple antennas at the transmitter and receiver. It requires the knowledge of channel state information (CSI) at transmitter, But know it is proved that we don't need perfect knowledge of CSI at transmitter[5]. This is done when $P$ rows out of $N$ rows are known in a matrix, a formula was derived which showed diversity order is directly proportional to $P$. It proved that partial CSI based scheme can be implemented instead of perfect CSI schemes[5]. Beamforming maximize capacity and also a significant array gain

In LTE beam forming uses similar approach as used in SM .It has a single codeword (no spatial multiplexing) and a single layer, implying that the layer mapping is transparent. LTE also supports more general non-codebook-based BM. Non-codebook-based BM in contrast to codebook-based BM has the terminal to make an estimate of the overall beam formed channel.. To enable this, LTE provides the possibility for the transmission of user equipment (UE)-specific reference symbols, transmitted using the same BM as thse user data, and enabled for the terminal to estimate the overall beam formed channel

\section{DOWNLINK SIMULATION PERFORMANCE ANALYSIS}

In this section the MIMO 4G technologies $(N T=N R=2)$ Throughput and PER results are represented using simulator and channel model. Channel synchronization and estimation are assumed to be perfect. Link speed basically combines modulation and coding rate. Our simulation generally support all modulations and coding rate. Peak data rate can be defined as

$\mathrm{D}=\mathrm{N}_{\mathrm{D}} \mathrm{N}_{\mathrm{b}} \mathrm{R}_{\mathrm{FEC}} \mathrm{R}_{\mathrm{STC}} / \mathrm{Ts}$

Where, $N_{D}$ is number of assigned data subcarriers to each user, $N_{b}$ is the bits per sub-carrier , $R_{F E C}$ is the forward error correction (FEC) coding rate , $\mathrm{R}_{\mathrm{STC}}$ is the space-time coding rate, $\mathrm{T}_{\mathrm{s}}$ is the OFDMA symbol duration. Using the above formula we calculated Peak data rate without error for different downlink mobile link speeds. perfect channel estimation and synchronization is assumed. The link throughput for each user is estimated from the PER as follows:

$\mathbf{R}=\mathbf{D}$ (1-PER) 
Where $\mathrm{D}$ represents the peak error free transmission rate for the chosen link speed( From above table) $R$ is Link throughput for each user,$D$ is Peak error -free transmission rate, PER is Packet error rate.

Table 2: shows the maximum peak data rate without error for WiMAX and LTE

\begin{tabular}{|c|c|c|}
\hline $\begin{array}{l}\text { MODE } \\
\text { (Link } \\
\text { speed) }\end{array}$ & $\begin{array}{l}\text { STBC Data Rate } \\
\text { (Mbps) } \\
\text { (Peak data rate } \\
\text { without error for } \\
\text { Mohilo WiMAX) }\end{array}$ & $\begin{array}{l}\text { STBC Data Rate } \\
\text { (Mbps) (Peak } \\
\text { data rate without } \\
\text { error for LTE) }\end{array}$ \\
\hline QPSK $1 / 2$ & 1.17 & 33.6 \\
\hline QPSK 3/4 & 1.75 & 50.4 \\
\hline 16 QAM & 2.33 & 67.2 \\
\hline 16 QAM & 3.50 & 100.8 \\
\hline 64 QAM & 3.50 & 100.8 \\
\hline 64 QAM & 4.66 & 134.4 \\
\hline 64 QAM & 5.25 & 151.2 \\
\hline
\end{tabular}

Table 2 shows the maximum of LTE, Downlink Mobile LTE Link-Speeds are calculated by

\section{$B S F=R b^{*} S c^{*} S^{*} M s^{*} B / T s$}

Where BSF is bits in sub frame, Rb is resource blocks, $\mathrm{Sc}$ is no of sub carriers, $\mathrm{S}$ is no of slots, Ms is no of Modulation Symbols, B represents no of bits and Ts is time slot.

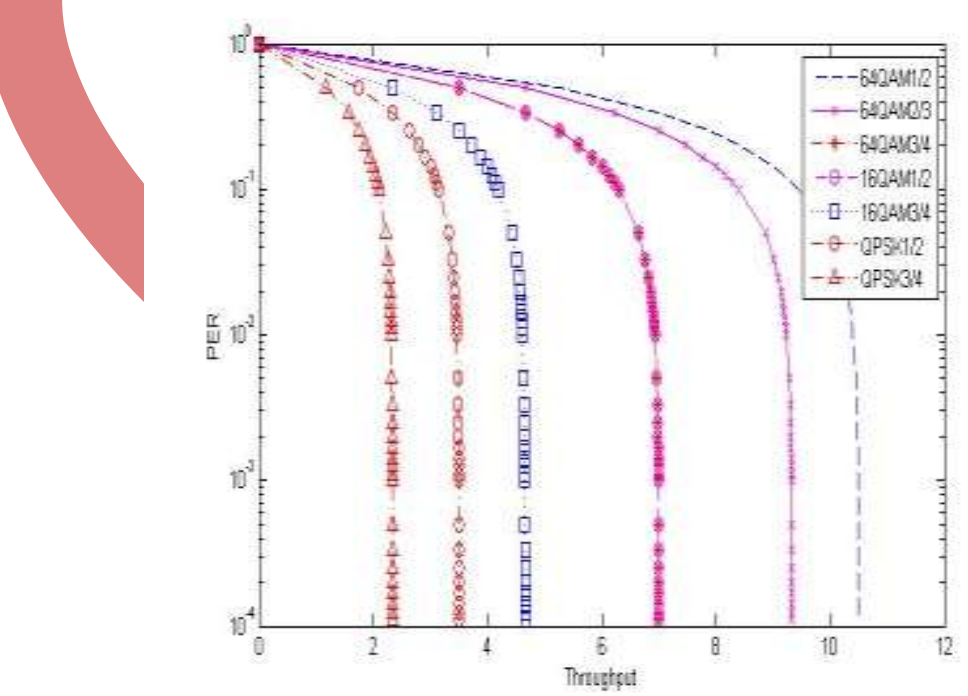

Fig 3: STBC $2 \times 2$ PER vs Throughput graph for Mobile WiMAX

The above graph compares the packet error rate performance for each of the modulation and channel coding schemes. It can be seen that the PER performance of the 64 QAM $3 / 4$.offers a significant improvement when compared with 16 QAM3/4. More specifically at a PER of $10^{-1}$.Also it can be interpreted from the above graph that $16 Q A M 1 / 2$ shows significant improvement over QPSK $1 / 2$ and 3/4.It signifies that TCP application requires higher modulation techniques and channel coding as it can tolerate high packet error rate as compared to UDP application. 


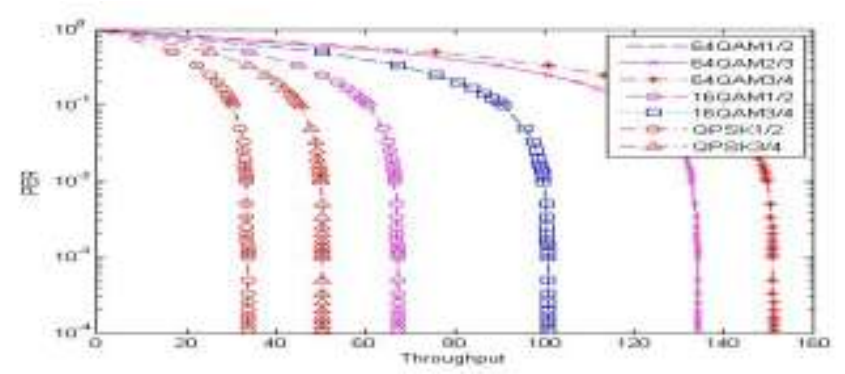

Fig 4:STBC $2 \times 2$ PER vs Throughput graph for LTE

In the above graph the modulation and coding technique 64QAM3/4 shows better performance as compared to 16 QAM $3 / 4$ . The modulation 16QAM3/4 shows better result than QPSK3/4. However, LTE requires higher throughput value to achieve desired PER as compared to WiMAX to achieve the preferred Quality of Service for a particular application.

\section{CONCLUSION}

The two basic layers of WiMAX and LTE that is PHY and MAC are discussed above which are necessary for improving overall QoS. Various smart antenna technique are also discussed in the paper, The detailed study of the throughput for a MIMO mobile WiMAX system and LTE under two different PHY PER QoS threshold. The MIMO channel was modeled using the 3GPP spatial channel model. The simulation is fully compliant with the IEEE standard. Throughput results were presented for a number of MIMO modes and all standardized link-speeds. The results clearly show that TCP applications achieve a higher throughput and operating range when compared with UDP applications. This occurs because of the higher PER threshold tolerated by the former protocol. Applications such as FTP and web-browsing can operate over larger distances than those sensitive to PER, such as VolP or video streaming. This is important in the cell planning process, where it is critical to support the QoS requirements of multiple applications running on multiple MSs.

\section{REFERENCES}

[1]. IEEE Std 802.16 TM-2004, "Part 16: Air interface for fixed broadband wireless access systems," Oct 2004.

[2]. IEEE Std 802.16e TM-2005, "Part 16: Air interface for fixed and mobile broadband wireless access systems," Feb. 2006

[3]. M. Alamouti, "A simple transmit diversity technique for wireless communications," IEEE JSAC, Vol. 16, No. 8, Oct.1998

[4] K.P Mar'e and B.T. Maharaj, "Performance Analysis of modern Space-Time codes

on a MIMO-WiMAX Platform"," IEEE International Conference on Wireless \& Mobile Computing, Networking \& Communication "MARCH, 2008

[5] Shimi Shilo, Anthony J. Weiss, Fellow, Amir Averbuch ,"Performance of Optimal Beamforming with Partial Channel Knowledge", IEEE TRANSACTIONS ON WIRELESS COMMUNICATIONS, VOL. 10, NO. 12, DECEMBER 2011.

[6]. J. G. Andrews, A. Ghosh and R. Muhamed, "Fundamentals of WiMAX, Understanding Broadband Wireless Networks," Prentice Hall, Feb 2007.

[7] Mai Tran, David Halls, Andrew Nix, Angela Doufexi and Mark Beach," Mobile WiMAX: MIMO Performance Analysis from a Quality of Service (QoS) Viewpoint "IEEE society COMMUNICATIONS WCNC 2009

[8] E. Dahlman et al., 3G Evolution: HSPA and LTE for Mobile Broadband, 2nd ed., Academic Press, 2008.

[9] 3GPP TS 36.211, "Evolved Universal Terrestrial Radio Access (E-UTRA); Physical Channels and Modulation," v. 8.9.0, Dec. 2009. 